Research Group: Food, Farms, and Firms

\title{
Intergenerational Transmission of Non-Communicable Chronic Diseases
}

\section{CATARINA GOULÃO AND AgUStin PEREZ-BARAHONA}




\title{
Intergenerational transmission of non-communicable chronic diseases
}

\author{
Catarina Goulão \\ Toulouse School of Economics (GREMAQ, INRA) \\ Agustín Pérez-Barahona* \\ INRA-AgroParisTech and École Polytechnique (France) \\ January 14, 2011
}

\begin{abstract}
We introduce a theoretical framework that contributes to the understanding of the non-communicable chronic diseases (NCDs) epidemics: even if NCDs are not "biologically" communicable, they may spread due to the transmission of unhealthy activities such as unhealthy diet, physical inactivity, and smoking. In particular, we study the intergenerational dimension of this mechanism. We find that, due to the "social" transmission of NCDs, agents choose lower health conditions and higher unhealthy activities than what is socially optimal. Taxes on unhealthy activities, that may subsidize health investments, can be used to restore the social optimum. Finally, we also observe that our model is consistent with the existence of regional asymmetries regarding the prevalence of obesity and NCDs.
\end{abstract}

Keywords: Health capital, Chronic diseases and obesity, Social transmission.

Journal of Economic Literature: H21; H23; I18.

* Corresponding author at: INRA-Économie publique, Avenue Lucien Brétignières, 78850 Thiverval Grignon, France; e-mail: agperez@grignon.inra.fr. 


\section{Introduction}

Non-communicable chronic diseases (NCDs) are "diseases or conditions (...) that affect individuals over an extensive period of time and for which there are no known causative agents that are transmitted from one affected individual to another." (Daar et al., 2007, p. 494). Despite being non-infectious NCDs are considered an epidemic because of their high prevalence rates. Indeed, according to Abegunde and Stanciole (2006), NCDs are increasing worldwide accounting for over half of the total deaths in the world, and $87 \%$ in high income countries. Moreover, the World Health Organization (WHO) points out that, even if NCDs have been commonly associated with the elderly of wealthy countries, at the present time NCDs are actually the major cause of death all over the world, except for Sub-Saharan Africa. In this regard, WHO (2008) estimates 35 million deaths each year due to NCDs. Besides death, NCDs also lead to substantial disabilitiy. Examples of NCDs are cardiovascular diseases, i.e., mainly heart disease and stroke, cancers, respiratory diseases, diabetes, and musculoskeletal disorders like osteoarthritis. In this paper, we provide an economic modelling of NCDs' epidemic mechanism enhancing its intergenerational determinants. Our contribution is also in view of understanding NCDs' economic implications and which policy instruments can be used to enhance welfare. Additionally, we attempt to contribute to the analysis of cross-country differences associated with the incidence of the epidemics of NCDs.

In general, epidemics have been widely studied from a medical perspective, paying special attention to the mathematical modeling of the epidemics of infectious diseases. A typical example in epidemiology is the well-known Compartmental Model (see for instance Kermack and McKendrick, 1927, 1932 and 1933; Bailey, 1975; Anderson and May, 1992; and Kuznetsov and Piccardi, 1994). However, there is a general agreement regarding the lack of explicit epidemics' economic modeling (Boucekkine et al., 2008 for a survey). Indeed, even if the epidemics mechanism has already been modeled for infectious diseases (Young, 2005; McDonald and Roberts, 2006; and Philipson, 2000), to our knowledge it has not yet been modeled for NCDs. Still, there are several empirical studies that focus on the relationship between economics and the prevalence of NCDs (see for instance Cumming, 1936; Lave and Seskin, 1971; Cropper, 1981; Mitchell, 1990; and Suhrcke et al., 2006). In contrast, from a 
theoretical point of view, the economic mechanisms behind NCDs' epidemics are still far from being understood.

The literature on the epidemics' economic modeling distinguishes between short and long-lived epidemics. A short-lived epidemic takes place in a very short period, after which the economy returns to its initial epidemiological environment. Therefore, short-lived epidemics are usually modeled as shocks on the initial conditions of the economy. Examples are the Black Death (Herlihy, 1997; and Hansen and Prescott, 2002) and the Spanish Flu (Boucekkine et al., 2008). On the other hand, long-lived epidemics are associated with long periods of disease and hence their effects cannot be reduced to a story of initial conditions shocks. As a result, the theory rationalizing this kind of epidemics turns out to be more complex. An example is HIV (see for instance Young, 2005; McDonald and Roberts, 2006; and Boucekkine et al., 2009). NCDs are indeed another example of long-lived epidemics. However, because they are non-infectious, the lack of knowledge about the dynamical mechanisms behind NCDs epidemics and economics calls for further theoretical effort. Our aim is precisely to contribute to the economic modeling of NCDs epidemics' dynamical mechanism.

The main causes of NCDs are genetics and age, as well as modifiable risk factors, such as unhealthy diet, physical inactivity, and smoking (WHO, 2005). The novelty of our approach is to provide a theoretical framework that incorporates the social transmission of the modifiable risk factors. That is to say, that even if NCDs are not infectious, we identify a transmission mechanism of its modifiable risk factors that contributes to explaining the epidemic dimension of NCDs. More precisely, we introduce an intergenerational transmission mechanism of the NCDs that being social is not biological.

We present an overlapping generations model in which agents live for three periods (childhood, adulthood and old age), and where the dynamics of the economy is based on health capital accumulation (Grossman, 1972). All economic decisions are made at adulthood and therefore parents decide upon their consumption levels and those of their children. More specifically, parents decide upon consumption levels of unhealthy goods (as for example salt, tobacco, saturated fat) and prevention ones (physical activity, medical care, etc.) that affect the level of health capital of the following period. The intergenerational 
NCDs' transmission is introduced through two different effects. The first assumes that children inherit their parents' health capital that is affected by their parents' choices of consumption of unhealthy and prevention goods. The second assumes that a child's probability of suffering from a NCD when old depends on her parents' health capital or, in other words, on her own health capital at the first period of life, also affected by her parents' choices. Assuming that individuals are not perfectly altruist, an externality arises since parents do not fully account for the effects of their choices on their children's health. Therefore the decentralized equilibrium is inferior to the social planner solution. We then analyse how policy instruments such as a tax on the unhealthy good, that may subsidize health investments, can be used to recover the social optimum. Our model also captures the existence of different development regimes linked to the presence of health thresholds. This provides therefore a reasoning for different regional NCDs prevalence rates. Moreover, we also study the role of health education or informational programs on the level of health capital, NCDs prevalence rates, and how it affects the optimal policy.

Our paper is organized as follows. In Section 2 we review the literature that provides evidence on NCDs social transmission in general and intergenerational transmission in particular. In Section 3 we present the model. Section 4 considers the decentralized solution and, in Section 5, we provide the welfare analysis. Finally, Section 6 concludes.

\section{Evidence on NCDs' social transmission}

Our economic modeling of NCDs' social transmission relies on three main assumptions. First, the modifiable risk factors such as unhealthy diet, physical inactivity, and smoking are socially transmissible. In particular, we assume an intergenerational transmission mechanism consisting of parents deciding upon their children's consumption of unhealthy and prevention goods. Second, parents' choices affect their children's health capital and, third, their children's probability of suffering from NCDs at old age. In this section we review the literature that provides evidence on these assumptions.

The idea that modifiable risk factors are socially transmitted is not new. 
For instance, Eisenberg (2004) analyses the effect of a user-friend's move or graduation on a young person's marijuana use, smoking, and binge drinking and finds that all decrease. Family ties and in particular those between parents and children are also very important in determining children's propensity in engaging in modifiable risk factors. Powell and Chaloupka (2005) find that teenagers are more likely to smoke if their parents do, although genetics could be playing a role. Overcoming the genetics determinant, Sacerdote (2007) uses data on adoptees to investigate the intergenerational transmission of modifiable risk factors on young adults offsprings and concludes that drinking behavior is transmitted equally well towards adoptees and nonadoptees. Regarding unhealthy eating and in particular disinhibited overeating, Francis et al. (2007) find that girls with both parents overweight had the highest increases in disinhibited overeating and BMI. Other authors have investigated to what extent mealtime environment and in particular parents' feeding styles contribute to the child's unhealthy eating. In this respect, Zeller et al. (2007) have shown that mothers of obese children experience more mealtime challenges and both parents experience less mealtime interactions. Additionally, obese mothers are more likely to offer their children food in response to emotional distress, use food as a form of reward, encourage children to eat beyond what was wanted, and control less high-fat foods intakes (Wardle et al., 2002). Still, there is an ongoing discussion concerning income classes' and race-related differences (Powers et al., 2006), as well as sex-related ones (Jahnke and Warschburger, 2008). All in all and with few exceptions, the medical and economic literature seems to agree on the intergenerational transmission of modifiable risk factors.

Evidence on parents' choices affecting their children's health capital is plentiful. Even if health capital is a complex concept (Grossman, 1972 and 2000) commonly used proxies are height (see, among others, Case and Paxson, 2008; Deaton, 2008; Deaton and Arora 2009; Bozolli et al., 2008; and Steckel, 1995 and 2008; Silventoinen, 2003) and body mass index (BMI) (see, for instance, Revicki and Israel, 1986; and WHO, 2004). Therefore, evidence on parents' choices affecting their children's height or BMI, as well as evidence on correlation between parents' and children's heights or BMIs give support to our assumption. In this regard, Chen and Li (2009) conclude that mother's education is an important determinant on a child's health, as measured by height- 
for-age-z-score. Additionally, the authors find the effect to be similar between adoptees and own-birth children putting therefore in evidence that behavior, just as genetics, is a channel of health transmission. Currie and Moretti (2003) also find that mothers attending college had a significant impact on children's health but Lindeboom et al. (2009) do not find any evidence of increasing the school leaving age of one year and offsprings' health. The latter authors remark that parents' education affecting children's health may be present only at sufficiently high education levels. Concerning obesity, a prominent example of parents' choices affecting children's health is tobacco consumption by pregnant women. Indeed, despite causing low birth-weights, it contributes to children's obesity at several ages (see for example Adams et al., 2005; Lake et al. 1997; Mamun et al., 2006; and Mendez et al., 2008). Moreover, there is a common agreement that children of obese parents are more likely to be obese at all ages, including adulthood (among others, Branca et al., 2007; and Abu-Rmeileh et al., 2008). Part of obesity's transmission is obviously due to genetics. Still, in the last 30 years obesity has doubled in the US (Cutler et al., 2003) and in many European countries it has increased threefold (WHO, 2009). Obviously such obesity prevalence increases cannot be supported by a similar genetic change (Hill and Peters, 1998). Accordingly, Bouchard (1996) estimates genetics to explain just between $25 \%$ and $40 \%$ of obesity rates increase, in accordance with Sacerdote's (2007) finding (46\%).

Finally, there is evidence of parent's choices affecting their children's probability of suffering from NCDs at old age (see, for instance, Osmond and Barker, 2000). Indeed, low birth weight is associated with increased probability of coronary heart diseases and diabetes in later life: the mechanism at stake is that fetal growth restriction, due amongst others to maternal smoking and unhealthy diet, may imply a reprograming of the metabolism. Barker and Clark (1997) and Godfrey and Barker (2000) survey this literature, and Victoria et al. (2008), Montgomery and Ekbom (2002), and Eriksson et al. (2001) provide further results. Also. van den Berg et al. (2007) using historical data for the Netherlands find evidence that early life exposure to the 1846-47 famine results in lower survival rates at old ages for men. 


\section{Setup}

Let us assume a discrete-time infinity-horizon economy populated by overlapping generations of agents living for three periods: childhood, adulthood, and old age. Time is indexed by $t=0,1,2, \ldots, \infty$, and all decisions are taken in the adult period of life. We also consider identical agents within each generation and no population growth (the size of each generation is normalized to 1 ). In this paper we assume that individuals might suffer from a NCD at the old age, and this will depend on their health capital.

Individual preferences are described by an expected lifetime utility function $U_{t}\left(c_{t}, v_{t}, h_{t+1}, \pi_{t}\right)$, where $\partial U_{t}(\cdot) / \partial c_{t}>0, \partial U_{t}(\cdot) / \partial v_{t}>0, \partial U_{t}(\cdot) / \partial h_{t+1}>0$, $\partial^{2} U_{t}(\cdot) / \partial c_{t}^{2}<0, \partial^{2} U_{t}(\cdot) / \partial v_{t}^{2}<0, \partial^{2} U_{t}(\cdot) / \partial h_{t+1}^{2}<0, \lim _{c_{t} \rightarrow 0} \partial U_{t}(\cdot) / \partial c_{t}=$ $+\infty, \lim _{v_{t} \rightarrow 0} \partial U_{t}(\cdot) / \partial v_{t}=+\infty$, and $\lim _{h_{t+1} \rightarrow 0} \partial U_{t}(\cdot) / \partial h_{t+1}=+\infty$. Agents care about consumption $c_{t}$ and unhealthy activities $v_{t}$, which encompass modifiable risk factors such as smoking, alcohol, consumption of fattening food and sugar-sweetened beverages. Moreover, following Grossman (1972, 2000), they are also concerned about their health capital when old $h_{t+1}$. We assume that individuals may suffer from a NCD at old age with a probability $\pi_{t}$. In particular, as in Blackburn and Cipriani (2002), we can consider the following function in order to get closed-form solutions:

$$
U_{t}\left(c_{t}, v_{t}, h_{t+1}, \pi_{t}\right)=\mu \ln c_{t}+\lambda \ln v_{t}+\left(1-\pi_{t}\right) \gamma \ln h_{t+1}+\pi_{t} \gamma(1-\phi) \ln h_{t+1},
$$

where $\mu>0$ and $\lambda>0$ represent, respectively, the weight that agents give to consumption and unhealthy activities, $\gamma>0$ stands for their concern about future health capital, and $\phi \in[0,1]$ represents the disutility of suffering from a NCD, which as a result of disease's morbidity and time loss because of treatment reduces utility driven from health capital. ${ }^{1}$

Consistently with the extensive medical literature on NCDs reviewed in Section 2 we assume that the probability of suffering from a NCD is a function of the inherited health capital, or in other words, the health capital at childhood (for an alternative economic modeling of early life conditions as determinants of health and economic outcomes see de la Croix and Licandro,

\footnotetext{
${ }^{1}$ Notice that our setup also allows for two extreme cases: mortal disease $(\phi=1)$, and negligible morbidity $(\phi=0)$.
} 
2007). Therefore $\pi_{t}=\pi\left(h_{t}\right)$, such that $\partial \pi\left(h_{t}\right) / \partial h_{t}<0, \lim _{h_{t} \rightarrow 0} \pi\left(h_{t}\right)=\pi_{H}$ and $\lim _{h_{t} \rightarrow \infty} \pi\left(h_{t}\right)=\pi_{L}$, with $0<\pi_{L}<\pi_{H}<1$.

Adult agents allocate their exogenous income $w_{t}$ among consumption, unhealthy activities, and health investments $m_{t}$ as medical care and physical activity. The corresponding budget constraint is

$$
w_{t}=c_{t}+v_{t}+m_{t}
$$

As in Grossman $(1972,2000)$, our model assumes that health capital accumulates over time. In particular, we consider the following law of motion:

$$
h_{t+1}=(1-\delta) h_{t}+\sigma m_{t}-\alpha v_{t}
$$

where $0<\delta<1$ and $\sigma, \alpha>0$. In this expression, $\delta$ represents the depreciation rate of health capital, $\sigma$ is the effectiveness of health investment, and $\alpha$ is the reduction of health conditions due to the unhealthy activities of individuals. Consistently with Grossman $(1972,2000)$, we assume that $\delta$ is not affected by illness. However, a disease induces utility loss through the parameter $\phi$ in equation $(1) .^{2}$

Equation (3) considers that agents may affect their health capital at the old age $h_{t+1}$ through health investment and unhealthy activities. However this also means that individual choices modify their children's inherited health capital as well. As a result the intergenerational transmission of NCDs occurs through two different channels: A direct channel, since parents' choices have a direct impact on their children's inherited health capital $\left(h_{t+1}\right)$, and an indirect channel, since parents' choices impact their children's probability of developing a NCD $\left(\pi\left(h_{t}\right)\right){ }^{3}$ Therefore, if individuals do not internalize these effects NCDs will spread to future generations. Thus, we model NCDs' epidemics based on a intergenerational transmission mechanism related to modifiable risk factors,

\footnotetext{
${ }^{2}$ Notice that, in our model, $h_{t+1}$ is the stock of health capital at the beginning of period $\mathrm{t}+1$, and the expected utility of health capital when old is thus given by $\left(1-\pi_{t}\right) \gamma \ln h_{t+1}+$ $\pi_{t} \gamma(1-\phi) \ln h_{t+1}$. An alternative modeling of the effect of the disease could consider a reduction of $h_{t+1}$ in equation (3). However, this would imply that children also inherit the disease, which is not appropriate for NCDs because they are non-communicable diseases by definition.

${ }^{3}$ Evidence supporting these two assumptions has been reviewed in Section 2.
} 
still acknowledging that NCDs are non-infectious diseases and, therefore, do not exhibit "biological" transmission among individuals. ${ }^{4}$

Finally, we also consider that agents may have a limited perception of the effect of the unhealthy choices on their own health and face a "perceived" law of motion of health capital

$$
h_{t+1}^{p}=(1-\delta) h_{t}+\sigma m_{t}-\epsilon \alpha v_{t},
$$

where $0<\epsilon<1$ represents agent's health information level. This assumption is consistent with evidence of poor nutritional knowledge not only of the population in general (Vereecken and Maes, 2010; Grimes et al., 2009; and Schwartz et al. 2005) but also of physicians (Flynn et al., 2003; and Makowske and Feinman, 2005). Additionally, there is evidence that greater nutritional knowledge is related to better nutrition (Kolodinsky et al., 2007; Pollard et al. 2010; and Vereecken and Maes, 2010). Still, when as is the case for NCDs the health-risk has mainly long-term effects, limited perception of health effects frequently arises (for a theoretical contribution see for instance Cremer et al., 2010; and for empirical evidence see for example Davison et al., 1991; Frankel et al., 1991; and Brownell et al., 2009; which relates sugar-sweetened beverages and obesity).

\section{Decentralized solution}

Let us first study the decentralized solution. Individuals choose consumption, unhealthy activities and health investments that maximize their utility subject to their budget constraint and the "perceived" law of motion of health capital. Knowing that, we can completely characterized the dynamics of the economy by taking the "true" law of motion of health capital (3).

\subsection{Individual behaviour}

Agents maximize $U_{t}\left(c_{t}, v_{t}, h_{t+1}^{p}, \pi_{t}\right)$ subject to (2), (4), $c_{t}>0, v_{t}>0, m_{t}>$ 0 and $h_{t}>0$, where $w_{t}$ and $h_{t}$ are taken as given. For a general utility

\footnotetext{
${ }^{4}$ We have already pointed out in Section 2 that genetics is not the main factor explaining the epidemics of NCDs (see for instance Hill and Peters, 1998; Bouchard, 1999; and Sacerdote, 2007).
} 
function, the corresponding first order conditions (FOCs) are summarized in the following expression: ${ }^{5}$

$$
\frac{\partial U_{t}}{\partial v_{t}}=\frac{\partial U_{t}}{\partial c_{t}}+\epsilon \alpha \frac{\partial U_{t}}{\partial h_{t+1}^{p}} .
$$

Considering the functional form (1), the optimal choices are

$$
\begin{gathered}
m_{t}=\frac{\sigma\left[\gamma(\sigma+\epsilon \gamma)\left(1-\phi \pi_{t}\right)+\epsilon \lambda \alpha\right] w_{t}-(1-\delta)[(\lambda+\mu) \sigma+\epsilon \mu \delta] h_{t}}{\sigma(\sigma+\epsilon \alpha)\left[\lambda+\mu+\gamma\left(1-\phi \pi_{t}\right)\right]}, \\
v_{t}=\frac{\lambda\left[(1-\delta) h_{t}+\sigma w_{t}\right]}{(\sigma+\epsilon \alpha)\left[\lambda+\mu+\gamma\left(1-\phi \pi_{t}\right)\right]}
\end{gathered}
$$

and

$$
c_{t}=\frac{\mu\left[(1-\delta) h_{t}+\sigma w_{t}\right]}{\sigma\left[\lambda+\mu+\gamma\left(1-\phi \pi_{t}\right)\right]} .
$$

From (6)-(8) we can observe that, all other things being equal, income $\left(w_{t}\right)$ has a positive effect on consumption, unhealthy activities and health investment. Moreover, greater inherited health conditions $\left(h_{t}\right)$ will increase consumption and unhealthy activities, but will decrease health investment: if inherited health conditions improve, investment in health capital is less needed. Regarding the probability of suffering from a NCD, the greater the probability the lower the value of old age for an individual. Therefore, $\pi_{t}$ has positive effect on consumption and unhealthy activities, but a negative one on health investment. Similarly, a greater disutility of NCD $(\phi)$ will reduce health investment, while consumption and unhealthy activities will rise. Finally, the more informed is an agent regarding the negative effect of her unhealthy behavior $(\epsilon)$ the higher the investment in health and the lower the consumption of unhealthy goods. ${ }^{6}$

\subsubsection{Dynamics}

The dynamics of our economy are completely characterized by the evolution of health capital, as given by the "true" law of motion. By substituting (6)-(8)

\footnotetext{
${ }^{5}$ Since agents inherit their current health conditions, our results would not change if $h_{t}$ is also introduced in the utility function.

${ }^{6}$ Notice that, due to the assumption of additive separable preferences (1), $\epsilon$ has not a direct effect on $c_{t}$. However, as we will see in Section 4.1.1, $\epsilon$ affects $c_{t}$ along both the short and long-run equilibrium.
} 
into (3), we get the corresponding transition function:

$$
h_{t+1}=\frac{\left[\gamma(\sigma+\epsilon \alpha)\left(1-\phi \pi\left(h_{t}\right)\right)-(1-\epsilon) \lambda \alpha\right]\left[(1-\delta) h_{t}+\sigma w\right]}{(\sigma+\epsilon \alpha)\left[\lambda+\mu+\gamma\left(1-\phi \pi\left(h_{t}\right)\right)\right]} \equiv \varphi\left(h_{t}\right),
$$

where income is assumed to be constant $\left(w_{t}=w\right)$ for the sake of simplicity. Since we are interested in positive interior solutions, Proposition 1 establishes sufficient condition for $\varphi\left(h_{t}\right)>0$.

Proposition 1 If NCDs' morbidity is high enough $(\phi>\tilde{\phi}$, where $\tilde{\phi}=1-$ $\lambda \alpha / \gamma \sigma)$ agents should be sufficiently informed about the effect of the unhealthy activities $\left(\epsilon>\tilde{\epsilon}\right.$, where $\left.\tilde{\epsilon}=\frac{\lambda \alpha-(1-\phi) \gamma \sigma}{\lambda \alpha+(1-\phi) \gamma \alpha}\right)$ and be sufficiently concerned about their future health conditions $(\gamma>\lambda \alpha / \sigma)$ to ensure positive health capital at old age $\left(\varphi\left(h_{t}\right)>0\right)$. Otherwise, $(\phi<\tilde{\phi})$, health capital at old age is always positive $\left(\varphi\left(h_{t}\right)>0\right)$.

Proof. From Equation (9) we find that $\varphi\left(h_{t}\right)>0 \Leftrightarrow \pi_{t}<\tilde{\pi}$, where $\tilde{\pi}=$ $\frac{1}{\phi}\left[1-\frac{(1-\epsilon) \lambda \alpha}{\gamma(\sigma+\epsilon \alpha)}\right]$. We consider all possible probabilities of disease $\left(0<\pi_{t}<1\right)$ and therefore restrict ourselves to the case $\tilde{\pi}>1$. Consequently, $\tilde{\pi}>1 \Rightarrow$ $\varphi\left(h_{t}\right)>0$. This sufficient condition can be rewritten as $\epsilon>\tilde{\epsilon}$, where $\tilde{\epsilon}$ is defined as in the proposition. Since $\epsilon$ is assumed to be strictly positive, it is easy to see from the definition of $\tilde{\epsilon}$ that the condition $\lambda \alpha-(1-\phi) \gamma \sigma<0$ implies $\tilde{\epsilon}<0 \Rightarrow \epsilon>\tilde{\epsilon} \Leftrightarrow \tilde{\pi}>1 \Rightarrow \varphi\left(h_{t}\right)>0$. Note also that $\lambda \alpha-(1-\phi) \gamma \sigma<0 \Leftrightarrow$ $\phi<\tilde{\phi}$, where $\tilde{\phi}$ is defined as in the proposition. Hence, if $\lambda \alpha-(1-\phi) \gamma \sigma<0$, $\phi<\tilde{\phi}$ is a sufficient condition for $\varphi\left(h_{t}\right)>0$. Otherwise, if $\lambda \alpha-(1-\phi) \gamma \sigma>0$ $(\Leftrightarrow \phi>\tilde{\phi})$ the sufficient condition becomes $\epsilon>\tilde{\epsilon}$ (notice that $\gamma>\lambda \alpha / \sigma$ is needed to well define $\tilde{\phi})$

Proposition 1 ensures positive health capital when NCDs' morbidity is high enough. In this case individuals should be sufficiently informed about unhealthy activities health effects and be concerned about their future health conditions.

In this paper we focus on the steady-state equilibrium $h^{*}$, which is defined as a fixed point of the transition function, i.e., $h^{*}=\varphi\left(h^{*}\right) .{ }^{7}$ One can easily verify that $h^{*}$ is stable (unstable) iff $\varphi^{\prime}\left(h^{*}\right)<1(>1)$. As in Azariadis (1996),

\footnotetext{
${ }^{7}$ Mathematically, this corresponds to a stationary point of the equilibrium difference equation (9).
} 
and Azariadis and Stachurski (2005), we can assume the following step function for the probability of disease in order to get further analytical results:

$$
\pi\left(h_{t}\right)=\left\{\begin{array}{lll}
\pi_{H} & \text { if } \quad h_{t}<h^{c} \\
\pi_{L} & \text { if } \quad h_{t} \geq h^{c}
\end{array}\right.
$$

where $h^{c}$ is an exogenous health threshold. According to this functional form, if the health conditions of an individual are low (high) enough $\left(h^{c}\right)$ the probability of suffering from a NCD will be high (low). The existence of health thresholds is well established in the medical literature. Indeed physicians make often use of thresholds to identify diseases and critical health conditions (see, for instance, Yuill and Miller, 2008: "cirrhosis in the liver may not result in a clinical effect until over $50 \%$ of the liver has been replaced by fibrous tissue"). Other well known examples are the thresholds for diabetes, blood pressure, obesity (BMI), etc.

Taking (10), the corresponding transition function is therefore composed by two branches given by

$$
\varphi\left(h_{t}\right)=\left\{\begin{array}{lll}
\frac{\left[\gamma(\sigma+\epsilon \alpha)\left(1-\phi \pi_{H}\right)-(1-\epsilon) \lambda \alpha\right]\left[(1-\delta) h_{t}+\sigma w\right]}{(\sigma+\epsilon \alpha)\left[\lambda+\mu+\gamma\left(1-\phi \pi_{H}\right)\right]} \equiv \varphi_{\pi_{H}}\left(h_{t}\right) & \text { if } & h_{t}<h^{c} \\
\frac{\left[\gamma(\sigma+\epsilon \alpha)\left(1-\phi \pi_{L}\right)-(1-\epsilon) \lambda \alpha\right]\left[(1-\delta) h_{t}+\sigma w\right]}{(\sigma+\epsilon \alpha)\left[\lambda+\mu+\gamma\left(1-\phi \pi_{L}\right)\right]} \equiv \varphi_{\pi_{L}}\left(h_{t}\right) & \text { if } & h_{t} \geq h^{c} .
\end{array}\right.
$$

In this case, we can prove that the dynamics of the model admits two stable steady-states:

Proposition 2 Assuming the functional form (10) and the conditions stablished in Proposition 1, there exist two stable steady-states $0<h_{\pi_{H}}^{*}<h^{c}<h_{\pi_{L}}^{*}$, given by

$$
h_{\pi_{i}}^{*}=\frac{\left[\sigma \psi_{\pi_{i}}-(1-\epsilon) \lambda \alpha\right] w}{\gamma \delta \psi_{\pi_{i}}+\sigma(\lambda+\mu)+\alpha[\lambda(1-\delta)+\epsilon(\mu+\lambda \delta)]},
$$

where $\psi_{\pi_{i}}=\gamma(\sigma+\epsilon \alpha)\left(1-\phi \pi_{i}\right)$ and $i=\{H, L\}$. (If instead $0<h_{\pi_{H}}^{*}<h_{\pi_{L}}^{*}<h^{c}$, the only steady-state is $h_{\pi_{H}}^{*}$. Finally, if $0<h^{c}<h_{\pi_{H}}^{*}<h_{\pi_{L}}^{*}$ the only steadystate is $h_{\pi_{L}}^{*}$ ).

Proof. It is easy to see that $0<\partial \varphi\left(h_{t}\right) / \partial h_{t}<1$ for all $h_{t}>0$, under the same conditions of Proposition 1. Therefore, assuming the step function (10), there exist two stable steady-states $h_{\pi_{i}}^{*}$ for $i=\{H, L\}$ if $h_{\pi_{H}}^{*}<h^{c}<h_{\pi_{L}}^{*}$ (notice that $h_{\pi_{H}}^{*}<h_{\pi_{L}}^{*}$ since $\left.\partial \varphi\left(h_{t}\right) / \partial \pi_{t}<0\right)$. The closed-forms of the steady-state values are provided by computing the fixed points of the transition function 
(11). Additionally one can easily check that if the health capital threshold is high enough so that $0<h_{\pi_{H}}^{*}<h_{\pi_{L}}^{*}<h^{c}$, the economy admits only a steadystate given by $h_{\pi_{H}}^{*}$. Otherwise, if the health capital threshold is low enough $0<h^{c}<h_{\pi_{H}}^{*}<h_{\pi_{L}}^{*}$ the only steady-state is $h_{\pi_{L}}^{*}$

Since $\varphi_{\pi_{i}}\left(h_{t}\right)$ is monotonically increasing in $h_{t}$, we can see that $h_{\pi_{i}}^{*}$ is positively affected by the income $(w)$, the effectiveness of health investment $(\sigma)$, the individual concern about future health capital $(\gamma)$, and agent's health information level $(\epsilon)$. However, a greater disability of NCDs $(\phi)$ will reduce the steady-state value of health capital. ${ }^{8}$

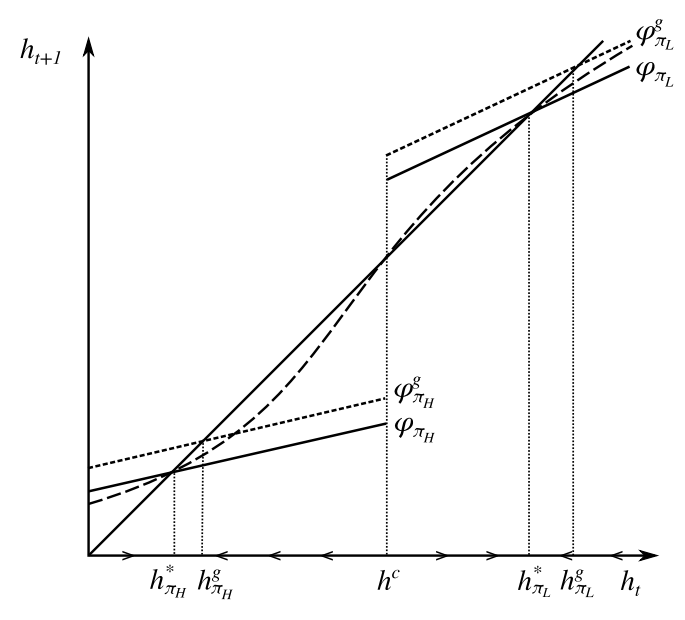

Figure 1: Dynamics

Figure 1 represents the two possible steady-states, assuming $0<h_{\pi_{H}}^{*}<$ $h^{c}<h_{\pi_{L}}^{*}$. The high (low) steady-state is associated with a high (low) level of health capital and a low (high) probability of suffering from a NCD. Indeed, if the initial health conditions are high (low) enough $\left(h_{0}>(<) h^{c}\right)$, the probability of being struck by a NCD is low (high). Therefore, agents will give a higher (lower) value to their old age. Consequently, this will induce a higher (lower) investment in health and lower (higher) consumption and unhealthy activities, and the economy will end up in the high (low) steady-state.

\footnotetext{
${ }^{8}$ Notice that if $\varphi\left(h_{t}\right)$ is concave and monotonically increasing in $h_{t}$, there is a unique stable steady-state $h^{*}$. In this case, we can also conclude from Equation (9) that $h^{*}$ is positively affected by $w, \sigma, \gamma$, and $\epsilon$. Moreover, the steady-state value is negatively affected by $\phi$ too.
} 
Finally, we can point out that multiple steady states may support the existence of regional asymmetries in what concerns obesity and NCDs in general. Even restricting ourselves to Europe, we can identify meaningful differences among countries. In 2005 in France, for example, $8 \%$ of men and 7\% of women are obese, contrasting enormously with $21 \%$ of men and $24 \%$ of obese women in the United Kingdom (WHO, 2009). Blanchflower and Oswald (2008) have also found evidence of country asymmetries with respect to hypertension.

\section{$5 \quad$ Welfare implications of NCDs}

In this section we show that a major consequence of the social transmission mechanism of NCDs is that individual choices are socially non-optimal. We calculate the social optimum and compare it with the decentralized solution characterized in the previous section. In addition to this analysis, Section 5.2 considers the golden rule defined by Chichilnisky et al. (1995). This allocation may be considered as a constrained social optimum in which the planner maximizes the aggregate surplus at the steady state, ignoring thus the transition process. It has the advantage of allowing us to provide further analytical results. Finally, in Section 5.3, we consider the implications of relaxing the assumption that individuals cannot affect their own probability of disease, i.e., we assume $\pi\left(h_{t+1}\right)$ instead of $\pi\left(h_{t}\right)$.

\subsection{Full-fledge forward-looking planner}

We characterize the social optimum by means of considering a full-fledge forward-looking planner, which maximizes a social welfare function $\beta^{-1} U_{-1}+$ $\sum_{t=0}^{\infty} \beta^{t} U_{t}\left(c_{t}, v_{t}, h_{t+1}, \pi_{t}\right)$ subject to $(2),(3), c_{t}>0, v_{t}>0, m_{t}>0$ and $h_{t}>0$, where $w$ and $h_{0}$ are taken as given, and $\beta \in(0,1)$ represents the inter-temporal discount rate. The corresponding Lagrangian is

$$
\mathcal{L}=\beta^{-1} U_{-1}+\sum_{t=0}^{\infty} \beta^{t}\left[U_{t}\left(c_{t}, v_{t}, h_{t+1}, \pi_{t}\right)+\iota_{t+1} \Omega_{t}\right],
$$

where $\Omega_{t}=(1-\delta) h_{t}+\sigma w-(\sigma+\alpha) v_{t}-\sigma c_{t}-h_{t+1}$ and $\iota_{t+1}>0$ is the Lagrangian multiplier (shadow price of health capital). The corresponding 
FOCs are summarized in

$$
\frac{\partial U_{t}}{\partial v_{t}}=\frac{\partial U_{t}}{\partial c_{t}}+\alpha\left\{\frac{\partial U_{t}}{\partial h_{t+1}}+\beta\left[\frac{\partial U_{t+1}}{\partial \pi_{t+1}} \frac{\partial \pi_{t+1}}{\partial h_{t+1}}+\iota_{t+2}(1-\delta)\right]\right\} .
$$

Comparing this expression with the FOC of the decentralized economy (5), we can conclude that the individual choices are socially non-optimal. Indeed, for $c_{t}$ and $h_{t+1}=h_{t+1}^{p}$ given, agents choose too much unhealthy activities than what is socially optimal (notice that $\partial^{2} U_{t}(\cdot) / \partial v_{t}^{2}<0$ ). Our model points out two sources of inefficiency. First, there is an intergenerational externality due to the social transmission of NCDs. Agents do not consider the direct effect of their individual choices on future health conditions, $\beta \iota_{t+2}(1-\delta)$. Moreover, they do not account for the indirect effect of their individual behaviour on the future generation through the probability of suffering from a NCD, $\beta \frac{\partial U_{t+1}}{\partial \pi_{t+1}} \frac{\partial \pi_{t+1}}{\partial h_{t+1}}$. Finally, a limited perception of the consequences of their unhealthy consumption $(\epsilon)$ will introduce an additional inefficiency. In fact, only if, simultaneously, there is no (social) transmission of NCDs ( $\delta \rightarrow 1$ and $\left.\frac{\partial \pi_{t+1}}{\partial h_{t+1}}=0\right)$ and agents have a high level of health information $(\epsilon \rightarrow 1)$, (5) and (13) coincide and the decentralized solution is thus socially optimal. Still, notice that the social transmission of NCDs generates inefficiency even under the case of low misperception $(\epsilon \rightarrow 1)$.

The previous result raises the question of how to implement the social optimum. In this paper we study the case of a tax on unhealthy activities. Real world examples encompass tobacco and alcohol taxation and the so called fat tax. A fat tax is a surcharge placed upon fattening foods and sugar-sweetened beverages with the aim of discouraging their consumption. Indeed the consumption of these products is considered a NCD modifiable risk factor since it contributes to an unhealthy diet and their increased consumption is associated with obesity epidemics (see for instance Vartanian et al., 2007; and Ludwig et al., 2001). Currently, fat taxes are under debate in several countries. In 2009, 33 states in the USA taxed soft drinks (Brownell et al., 2009). Moreover, France is considering plans to impose a fat tax on junk food (see IGF, 2008; Bonnet et al., 2009; and Allais et al., 2010). Non surprisedly, part of the food and beverage industry strongly criticizes this policy due to sales reduction (see, for instance, Brownell et al., 2009; and Vartanian et al., 2007). One of their main arguments considers that fat taxes are against individual freedom: contrary to tobacco and alcohol, the consumption of fattening food 
and soft drinks does not involve negative externalities as second hand smoke and drunk driving accidents (Rudd, 2009). Nevertheless, authors as Brownell et al. (2009) and Finkelstein et al. (2009) have identified external effects due to the rise of obesity-related medical expenditures. In this regard, our paper contributes to this literature by pointing out another external effect (an intergenerational externality) related to the social transmission of NCDs that may justify the usage of this kind of taxes. However, several drawbacks of fat taxes have been already identified in the litterature. A commonly-raised problem is the regressive nature of the policy (Allais et al., 2010). Nevertheless, several authors (see for instance, Brownell et al., 2009; Jacobson and Brownell, 2000; and Rudd, 2009) point out that such a problem is minimized if the revenues of the fat taxes are used in the benefit of the poor. In this direction, despite that redistribution concerns are beyond the scope of our paper (for an analytical dynamic setup of income distribution under epidemics, see Boucekkine and Laffargue, 2010), we model fat taxes where the corresponding revenues are used to finance healthy activities.

Let us consider the decentralized problem with a tax $\left(\tau_{t}\right)$ on unhealthy activities. We use the corresponding tax revenues to subsidize $\left(s_{t}\right)$ the healthy activities (see for instance Cremer et al., 2010, for another theoretical contribution). Individuals maximize $U_{t}\left(c_{t}, v_{t}, h_{t+1}^{p}, \pi_{t}\right)$ subject to (4) and the modified budget constraint

$$
w=c_{t}+\left(1-s_{t}\right) m_{t}+\left(1+\tau_{t}\right) v_{t}
$$

taking $s_{t}$ and $\tau_{t}$ as given. Finally, at the equilibrium, $s_{t} m_{t}=\tau_{t} v_{t}$ for all $t \geq 0$. The corresponding FOC is

$$
\frac{\partial U_{t}}{\partial v_{t}}=\frac{\partial U_{t}}{\partial c_{t}}+\left(\sigma \frac{\tau_{t}}{1-s_{t}}+\epsilon \alpha\right) \frac{\partial U_{t}}{\partial h_{t+1}^{p}},
$$

Since at the social optimum $h_{t+1}=h_{t+1}^{p}$, we get the trajectory for the optimal policy by equating (13) and (15):

$$
\frac{\tau_{t}}{1-s_{t}}=\frac{\alpha}{\sigma}\left\{(1-\epsilon)+\beta\left[\frac{\frac{\partial U_{t+1}}{\partial \pi_{t+1}} \frac{\partial \pi_{t+1}}{\partial h_{t+1}}+(1-\delta) \iota_{t+2}}{\frac{\partial U_{t}}{\partial h_{t+1}}}\right]\right\} .
$$

As it is clear from the previous expression, this optimal policy considers the two sources of inefficiency described before, i.e., the intergenerational externality (direct and indirect effects) and the limited perception of the agents. Indeed, 
as one can expect, in absence of transmission mechanism $\left(\delta \rightarrow 1\right.$ and $\left.\frac{\partial \pi_{t+1}}{\partial h_{t+1}}=0\right)$ and misperception $(\epsilon \rightarrow 1)$ the optimal tax and subsidy vanish. ${ }^{9}$

\subsection{Golden rule}

Let us now consider the golden rule allocation defined in Chichilnisky et al. (1995). As in John and Peccenino (1994), this case may be interpreted as a particular social planner problem where we maximize the aggregate utility at the steady-state. The main advantage of this solution is that it allows us to provide further analytical results regarding social welfare.

The social planner maximizes $U(c, v, h, \pi)$ subject to $(2)$ and (3) at the steady-state, $c>0, v>0, m>0$ and $h>0$. The corresponding FOC is provided by

$$
\frac{\partial U}{\partial v}=\frac{\partial U}{\partial c}+\frac{\alpha}{\delta}\left(\frac{\partial U}{\partial h}+\frac{\partial U}{\partial \pi} \frac{\partial \pi}{\partial h}\right)
$$

Similar to the case of full-fledge forward-looking planner, the FOC of the decentralized economy (5), at the steady-state, does not coincide with equation (17) because of the intergeneration transmission of NCDs and the misperception problem. As before, we can implement the golden rule by means of a tax $(\tau)$ on the unhealthy consumption and a subsidy $(s)$ on the healthy activities. Taking $h_{t+1}=h_{t+1}^{p}$, we get the corresponding policy by equating (15) at the steady state with (17):

$$
\frac{\tau}{1-s}=\frac{\alpha}{\sigma}\left[\left(\frac{1}{\delta}-\epsilon\right)+\frac{1}{\delta} \frac{\frac{\partial U}{\partial \pi} \frac{\partial \pi}{\partial h}}{\frac{\partial U}{\partial h}}\right] .
$$

As it is clear from (17) and (18), under a high level of health information $(\epsilon \rightarrow 1)$ and no intergenerational transmission $\left(\delta \rightarrow 1\right.$ and $\left.\frac{\partial \pi}{\partial h}=0\right)$ both FOCs coincide and, therefore, tax and subsidy become zero (notice that, at the equilibrium, $s m=\tau v)$.

\footnotetext{
${ }^{9}$ Notice that the optimal solution can be also decentralized by means of a tax on unhealthy activities and a lump-sum transfer $T_{t}$ of the corresponding tax revenues to the agents (i.e., the modified budget constraint would be $w+T_{t}=c_{t}+m_{t}+\left(1+\tau_{t}\right) v_{t}$ and, at the equilibrium, $\left.T_{t}=\tau_{t} v_{t}\right)$. In our model, this case is equivalent to consider $s_{t}=0$.
} 


\subsubsection{Golden rule in our setting}

Let us consider the functional forms (1) and (10). Taking (2) and (3) at the steady-state, and the equation (17), the golden rule values for health capital and unhealthy activities are respectively:

$$
h_{\pi_{i}}^{g}=\frac{\gamma \sigma\left(1-\phi \pi_{i}\right) w}{\delta\left[(\lambda+\mu)+\gamma\left(1-\phi \pi_{i}\right)\right]}
$$

and

$$
v_{\pi_{i}}^{g}=\frac{\lambda \sigma w}{(\sigma+\alpha)\left[(\lambda+\mu)+\gamma\left(1-\phi \pi_{i}\right)\right]},
$$

for $i=\{H, L\} .{ }^{10}$ As it is clear from Proposition 2, the individual choices are different than the golden rule allocation. Indeed, we can claim that due to the transmission mechanism of NCDs and the misperception problem agents will choose too many unhealthy activities and too little health capital:

Proposition 3 At the steady-state, under Proposition 2, individuals choose higher quantity of unhealthy activities and lower health capital than the golden rule allocation.

Proof. Taking (7) at the steady state and (20), it is easy to see that $v_{\pi_{i}}^{*}>v_{\pi_{i}}^{g}$ since $\epsilon<1$ and, from Proposition $2, h_{\pi_{i}}^{*}>0$, for $i=\{H, L\}$. Let us now prove that $h_{\pi_{i}}^{*}<h_{\pi_{i}}^{g}$, for $i=\{H, L\}$. To do that it is sufficient to prove that $\varphi_{\pi_{i}}\left(h_{t}\right)<\varphi_{\pi_{i}}^{g}\left(h_{t}\right)$, for all $h_{t}>0$, where $\varphi_{\pi_{i}}^{g}\left(h_{t}\right)=(1-\delta) h_{t}+\sigma m_{\pi_{i}}^{g}-\alpha v_{\pi_{i}}^{g}$ (the corresponding $\pi_{i}$-branch for the golden rule problem). From (2) and (3) in the golden rule problem, it is easy to get $m_{\pi_{i}}^{g}=\frac{\left[\lambda \alpha+\gamma(\sigma+\alpha)\left(1-\phi \pi_{i}\right)\right] w}{(\sigma+\alpha)\left[(\lambda+\mu)+\gamma\left(1-\phi \pi_{i}\right)\right]}>0$. Therefore, taking $(20), \varphi_{\pi_{i}}^{g}\left(h_{t}\right)=\frac{\left[(\lambda+\mu)+\gamma\left(1-\phi \pi_{i}\right)\right](1-\delta) h_{t}+\gamma \sigma\left(1-\phi \pi_{i}\right) w}{(\lambda+\mu)+\gamma\left(1-\phi \pi_{i}\right)}$. Rearranging terms in (11) we get $\varphi_{\pi_{i}}\left(h_{t}\right)=\frac{\left[\gamma\left(1-\phi \pi_{i}\right)-\left(\frac{1-\epsilon}{\sigma+\epsilon \alpha}\right) \lambda \alpha\right]\left[(1-\delta) h_{t}+\sigma w\right]}{(\lambda+\mu)+\gamma\left(1-\phi \pi_{i}\right)}$. Finally, by comparing these two expressions, it is easy to conclude that $\varphi_{\pi_{i}}\left(h_{t}\right)<\varphi_{\pi_{i}}^{g}\left(h_{t}\right)$, for all $h_{t}>0$

Furthermore, from equation (2), we can also conclude that agents choose too much consumption $\left(c_{\pi_{i}}^{*}>c_{\pi_{i}}^{g}\right)$ and too little health investment $\left(m_{\pi_{i}}^{*}<\right.$

\footnotetext{
${ }^{10}$ Without lost of generality, this paper focuses on the case of a golden rule allocation associated to each steady-state established in Proposition 2. As it is clear from Figure 1, multiplicity arises if $h_{\pi_{H}}^{g}<h^{c}<h_{\pi_{L}}^{g}$ (notice that $h_{\pi_{H}}^{g}<h_{\pi_{L}}^{g}$ since $\partial h_{\pi_{i}}^{g} / \partial \pi_{i}<0$ ). However, if $h^{c}<h_{\pi_{H}}^{g}$, the golden rule allocation is unique and given by $h^{g}=h_{\pi_{L}}^{g}$. In this case, it is easy to see that the results provided in this section remain the same.
} 
$\left.m_{\pi_{i}}^{g}\right)$ at the steady-state. From (18) and assuming the step function (10), the corresponding policy to implement the golden rule is now given by

$$
\frac{\tau_{\pi_{i}}}{1-s_{\pi_{i}}}=\frac{\alpha}{\sigma}\left(\frac{1}{\delta}-\epsilon\right)
$$

Moreover, we can establish the following proposition:

Proposition 4 In our setting, the golden rule allocation can be decentralized by means of a tax $\tau_{\pi_{i}}$ on the unhealthy consumption, and a subsidy $s_{\pi_{i}}$ on the healthy activities, which is financed by the $\tau_{\pi_{i}}$ revenues. The corresponding closed forms are given by

$$
\tau_{\pi_{i}}=\frac{\tilde{\tau}}{1+\frac{v_{\pi_{i}}^{g}}{m_{\pi_{i}}^{g}} \tilde{\tau}}
$$

and

$$
s_{\pi_{i}}=\tau_{\pi_{i}} \frac{v_{\pi_{i}}^{g}}{m_{\pi_{i}}^{g}}
$$

where $\tilde{\tau}=\frac{\alpha}{\sigma}\left(\frac{1}{\delta}-\epsilon\right)$ and $\frac{v_{\pi_{i}}^{g}}{m_{\pi_{i}}^{g}}=\frac{\lambda \sigma}{\lambda \alpha+\gamma\left(1-\phi \pi_{i}\right)(\sigma+\alpha)}$.

Proof. From (21) we know that $\frac{\tau_{\pi_{i}}}{1-s_{\pi_{i}}}$ decentralizes the golden rule allocation. Since $s_{\pi_{i}} m_{\pi_{i}}^{g}=\tau_{\pi_{i}} v_{\pi_{i}}^{g}$, we get (22) and (23). Finally, from (20) and the formula of $m_{\pi_{i}}^{g}$ (see the proof of Proposition 3) we obtain the expression for $\frac{v_{\pi_{i}}^{g}}{m_{\pi_{i}}^{g}}$

Finally, from this proposition we can also conclude that the greater the misperception problem (i.e, the lower $\epsilon$ ) the greater should be the tax and the subsidy. This result points out the importance of considering agent's health information level to study the economic impact of this kind of policies. In this regard, Allais et al. (2010) have predicted little effect of fat taxes on French consumers. However, they also recognise that their study does not include the effect of informational programs. Indeed, Pollard et al. (2009) concluded about the substantial effectiveness of the "Go for $2 \& 5$ " (2 fruits and 5 vegetables a day) campaign in Australia. Moreover, Bonnet et. al (2009), using the same data base as Allais et al. (2010), showed that the estimated price elasticities of individual consumption are significant and may justify a tax on high density and cheap energy categories of food such as junk food as effective policy to reduce obesity and overweight. ${ }^{11}$ For further empirical results in the same direction see, for instance, Mytton et al. (2007) and Epstein et al. (2007).

\footnotetext{
${ }^{11}$ Among other things, they found that a $10 \%$ increase of junk food prices together with a $10 \%$ reduction of fruits and vegetables prices would induce a reduction of the proportion of
} 


\subsection{Further analytical results}

As we have observed in Section 1, our set-up considers $\pi_{t}=\pi\left(h_{t}\right)$. Still, it may be reasonably argued that individuals also affect their own probability of disease. For this reason, let us now study the case $\pi_{t}=\pi\left(h_{t+1}\right)$ : agents may modify their own probability of suffering from a NCD, which is determined just before arriving to the old-age. The FOC corresponding to the decentralized solution is given by

$$
\frac{\partial U_{t}}{\partial v_{t}}=\frac{\partial U_{t}}{\partial c_{t}}+\epsilon \alpha\left(\frac{\partial U_{t}}{\partial h_{t+1}^{p}}+\frac{\partial U_{t}}{\partial \pi_{t}} \frac{\partial \pi_{t}}{\partial h_{t+1}^{p}}\right) .
$$

Comparing this expression with (5), one can observe that agents consider the effect of the individual choices on the probability of suffering from a NCD because they can modify their own $\pi_{t}$. Let us now study the social optimum. The FOC corresponding to the full-fledge social planner is given by

$$
\frac{\partial U_{t}}{\partial v_{t}}=\frac{\partial U_{t}}{\partial c_{t}}+\alpha\left[\frac{\partial U_{t}}{\partial h_{t+1}}+\frac{\partial U_{t}}{\partial \pi_{t}} \frac{\partial \pi_{t}}{\partial h_{t+1}}+\beta \xi_{t+2}(1-\delta)\right],
$$

where $\xi_{t+2}>0$ is the corresponding Lagrangian multiplier. From (24) and (25), taking $h_{t+1}=h_{t+1}^{p}$, it is easy to see that part of the effect of the social transmission of NCDs is internalized. Indeed, the indirect effect of the individual behaviour on the probability of suffering from a NCD partially vanishes if agents can modify $\pi_{t}$. However, agents completely neglect the direct effect on the future generations, $\beta \xi_{t+2}(1-\delta)$. Moreover, as before, the misperception problem $(\epsilon)$ adds an additional source of inefficiency.

Similar to the previous case, we can decentralized the social planner solution by means of a tax $\left(\tau_{t}\right)$ on the unhealthy activities, that we use to subsidize $\left(s_{t}\right)$ the healthy activities. The individuals' FOC is now given by

$$
\frac{\partial U_{t}}{\partial v_{t}}=\frac{\partial U_{t}}{\partial c_{t}}+\left(\sigma \frac{\tau_{t}}{1-s_{t}}+\epsilon \alpha\right)\left(\frac{\partial U_{t}}{\partial h_{t+1}^{p}}+\frac{\partial U_{t}}{\partial \pi_{t}} \frac{\partial \pi_{t}}{\partial h_{t+1}^{p}}\right),
$$

and, taking $h_{t+1}=h_{t+1}^{p}$, the optimal policy is provided by equating (25) and $(26)$ :

$$
\frac{\tau_{t}}{1-s_{t}}=\frac{\alpha}{\sigma}\left[(1-\epsilon)+\beta(1-\delta)\left(\frac{\xi_{t+2}}{\frac{\partial U_{t}}{\partial h_{t+1}}+\frac{\partial U_{t}}{\partial \pi_{t}} \frac{\partial \pi_{t}}{\partial h_{t+1}}}\right)\right] .
$$

overweight (children: -33:64\%; adult males: $-8.78 \%$; and adult females: $-11.65 \%$ ) and obese (children: $-30.88 \%$; adult males: $-11.13 \%$; and adult females: $-20.61 \%$ ). 
Let us compare this expression with Equation (16). In the case of $\pi_{t}=\pi\left(h_{t}\right)$, the optimal policy includes, in addition to the misperception problem, both the direct and indirect effect of the individual choices on future generations' health. However, if agents can modify their own probability of suffering from a NCD, we only need to correct the direct effect and $\epsilon$. Moreover, from (27) it is clear that $\frac{\partial U_{t}}{\partial \pi_{t}} \frac{\partial \pi_{t}}{\partial h_{t+1}}$ allows us to reduce the optimal policy (for instance, a lower fat tax in the case of a lump-sum transfer). Finally, under a high level of health information $(\epsilon \rightarrow 1),(24)$ and (25) coincide under absence of transmission mechanism of NCDs $(\delta \rightarrow 1)$. Therefore, the corresponding tax and subsidy vanish. ${ }^{12}$

\section{Concluding remarks}

We have developed a model that rationalizes the "social" transmission mechanism behind NCDs' epidemics: the transmission of modifiable risk factors results in a intergenerational externality. Such externality arises through two different effects: a direct effect because parents affect the inherited health capital of their children, and an indirect effect because they affect the probability of their children suffering from a chronic disease when old. Parents are not fully altruistic and when they make their choices they do not entirely account for the impact of their choices on their children's health. Consequently, the negative externality results in a lower health capital level and a higher level of unhealthy activities at the descentralized equilibrium, which contrasted to the optimal choice. Taxes on unhealthy activities, where the corresponding revenues are used to subsidized health investments (Cremer et al., 2010), can be used to restore socially optimal health capital and unhealthy activities' levels. Only when the two effects are neutralized is the externality absent. Still, even in the absence of externality there is room for public intervention if individuals

\footnotetext{
${ }^{12}$ Notice that, when $\pi_{t}=\pi\left(h_{t}\right)$, we have assumed a step function (10) in order to provide closed-form solutions (see sections 4.1 .1 and 5.2.1). Indeed, this functional form may be considered as a discretized version of a convex-concave $\pi\left(h_{t}\right)$ (in Figure 1, the convex-concave broken line corresponds to a convex-concave $\psi\left(h_{t}\right)$ ). However, a step-simplification is not possible if $\pi_{t}=\pi\left(h_{t+1}\right)$ : the function must be differentiable in its whole domain. In this case, we may directly consider a convex-concave function like $\pi\left(h_{t}\right)=\left(\pi_{L}-\pi_{H}\right) \frac{h_{t+1}^{2}}{1+h_{t+1}^{2}}+\pi_{H}$. Nevertheless, the analysis would be restricted to implicit functions and numerical results.
} 
are not well informed about unhealthy activities health effects. Finally, we also show that our model is consistent with the existence of international/regional asymmetries regarding the prevalence of obesity and NCDs.

Several remarks can be made with regard to our modelization. Since our paper focuses on the intergenerational transmission of NCDs, we have considered a simple set-up that incorporates a social transmission mechanism of the disease. However, we could extend our framework by adding other effects behind epidemics. Among them, would be an endogenous income effect. Indeed, Boucekkine et al. (2009) have already studied the interaction between epidemics and income within the context of communicable diseases such as HIV/AIDS and malaria. In particular, they empirically found a significant effect of this kind of epidemics on educational choices and wages. Following de la Croix and Doepke (2003), one could endogenize income in our set-up as being a function of human capital $w\left(\tilde{h}_{t}\right)$ : NCDs would affect income through agent's educational choices. Finally, another simplification of our framework is the absence of savings: since in our model health is already an inter-temporal choice variable we did not include physical capital (savings) for simplicity. Therefore, one could incorporate savings as a technical extension of our paper. In this regard the approach introduced by Mariani et al. (2010) might provide a fruitful possibility.

\section{References}

Abegunde D. and A. Stanciole (2006) "An estimation of the economic impact of chronic noncommunicable disease in selected countries" World Health Organization Department of Chronic Diseases and Health Promotion (CHP). Available from http://www.who.int/chp

Abu-Rmeileh N., C. Hart, A. McConnachie, M. Upton, M. Lean and G. Watt (2008) "Contribution of Midparental BMI and Other Determinants of Obesity in Adult Offspring" Obesity 16 (6), 1388-1393.

Adams A., H. Harvey and R. Prince (2005) "Association of maternal smoking with overweight at age $3 \mathrm{y}$ in American Indian children" The American Journal of Clinical Nutrition 82, 393-398. 
Allais O., P. Bertail and V. Nichèle (2010) "The Effects of a Fat Tax on French Households' Purchases: A Nutritional Approach" American Journal of Agricultural Economics 92 (1), 228-245.

Anderson R. and R. May (1992) Infectious Diseases of Humans, Oxford University Press: New York.

Azariadis C. (1996) "The Economics of Poverty Traps Part One: Complete Markets" Journal of Economic Growth 1, 449-486.

Azariadis C. and J., Stachurski (2005) "Poverty traps" in Handbook of Economic Growth by Aghion P. and S. Durlauf, Eds., Elsevier B.V.: Amsterdam, 1A, 295-384.

Bailey N. (1975) The mathematical Theory of Infectious Diseases (2nd edition). Charles Griffin and co. Ltd.: London.

Barker D. and P. Clark (1997) "Fetal undernutrition and disease in later life" Reviews of Reproduction 2, 105-112.

van den Berg G.J., M. Lindeboom and F. Portrait (2007) "Long-run longevity effects of a nutritional shock early in life: the Dutch potato famine of 18461847” IZA DP 3123.

Blackburn K. and G. Cipriani (2002) "A model of longevity, fertility and growth" Journal of Economic Dynamics and Control 26, 187-204.

Blanchflower D.G. and A.J. Oswald (2008) "Hypertension and happiness across nations" Journal of Health Economics 27(2), 218-233.

Bonnet C., P. Dubois and V. Orozco (2009) "Food Consumption and Obesity in France: Identification of Casual Effects and Price Elasticities" Mimeo.

Bouchard C. (1996) "Genetics of obesity in humans: current issues" in The origins and consequences of obesity by Chardwick D.J. and G.C. Cardew, Eds., Wiley: Chichester, 108-117 (CIBA Foundation Symposium 201).

Boucekkine R., R. Desbordes and H. Latzer (2009) "How do epidemics induce behavioral changes?" Journal of Economic Growth 14, 233-264. 
Boucekkine R., B. Diene and T. Azomahou (2008) "Growth economics of epidemics: a survey of the theory" Mathematical Population Studies 15, 1-26.

Boucekkine R. and J. Laffargue (2010) "On the distributional consequences of epidemics" Journal of Economic Dynamics and Control 34, 231-245.

Bozzoli C., A. Deaton and C. Quintana-Domeque (2008) "Adult height and childhood disease" FEDEA Working Papers 2008-25.

Branca F., H. Nikogosian and T. Lobstein (2007) The challenge of obesity in the WHO European Region and the strategies for response, Copenhagen: WHO Press, World Health Organization.

Brownell K., T. Farley, W. Willett, B. Popkin, F. Chaloupka, J. Thompson and D. Ludwig (2009) "The public health and economic benefits of taxing sugarsweetened beverages" New England Journal of Medicine 361 (16), 1599-1605.

Case A. and C. Paxson (2008) "Height, Health and Cognitive Function at Older Ages" American Economic Review 98 (2), 463-67.

Chen Y. and H. Li (2009) "Mother's education and child health: Is there a nurturing effect?" Journal of Health Economics 28, 413-426.

Chichilnisky G., G. Heal and A. Beltratti (1995) "The green golden rule" Economics Letters 49, 175-179.

Cremer H., P. De Donder, D. Maldonado and P. Pestieau (2010) "Taxing sin goods and subsidizing health care" Scandinavian Journal of Economics, forthcoming.

de la Croix D., M. Doepke (2003) "Inequality and growth: why differential fertility matters" American Economic Review 93 (4), 1091-1113.

de la Croix D. and O. Licandro, 2007. The child is father of the man: implications for the demographic transition. CORE DP 2007-72, Catholic university of Louvain (Belgium).

Cropper M. (1981) "Measuring the benefits from reduced morbidity" American Economic Review 71 (2), 235-240. 
Cumming H. (1936) "Chronic disease as a public health problem" The Milbank Memorial Fund Quarterly 14 (2), 125-131.

Currie J. and E. Moretti (2003) "Mother's education and the intergenerational transmission of human capital: evidence from college openings" Quarterly Journal of Economics 118, 1495-1532.

Cutler D., E. Glaeser and J. Shapiro (2003) "Why Have Americans Become More Obese?" Journal of Economic Perspectives, 17 (3), 93-118.

Daar A., P. Singer, D. Persad, S. Pramming, D. Matthews, R. Beaglehole, A. Bernstein, L. Borysiewicz, S. Colagiuri, N. Ganguly, R. Glass, D. Finegood, J. Koplan, E. Nabel, G. Sarna, N. Sarrafzadegan, R. Smith, D. Yach and J. Bell (2007) "Grand challenges in chronic non-communicable diseases. The top 20 policy and research priorities for conditions such as diabetes, stroke and heart disease" Nature 450 (22), 494-496.

Davison C, G. Smith and S. Frankel (1991) "Lay epidemiology and the prevention paradox - the implication of coronary candidacy for health education" Sociology of Health \& Illness 13, 1-19.

Deaton A. (2008) "Income, health, and well-being around the world: Evidence from the Gallup world pool" Journal of Economic Perspectives 22, 53-72.

Deaton A. and R. Arora (2009) "Life at the top: The benefits of height" Economics and Human Biology 7, 133-136.

Dietz W. (1998) "Health consequences of obesity in youth: childhood predictors of adult disease" Pediatricsm 101, 518-525.

Eisenberg D. (2004) "Peer effects for adolescent substance use: do they really exist?" Working Paper, University of California - Berkeley School of Public Health.

Epstein L., K. Dearing, R. Paluch, J. Roemmich, D. Cho (2007) "Price and maternal obesity influence purchasing of low- and high-energy-dense foods" American Journal of Clinical Nutrition 86, 914-922. 
Eriksson J., T. Forsén, J. Tuomilehto, C. Osmond and D. Barker (2001) "Early growth and coronar y heart disease in later life: longitudinal study" British Medical Journal 322 (21), 949-953.

Finkelstein E., J. Trogdon, J. Cohen and W. Dietz (2009) "Annual Medical Spending Attributable To Obesity: Payer-And Service-Specific Estimates" Health Affairs 28, 822-831.

Flynn M., C. Sciamanna and K. Vigilante (2003) "Inadequate physician knowledge of the effects of diet on blood lipids and lipoproteins" Nutrition Journal $2: 19$.

Francis L., A. Ventura, M. Marini, L. Birch (2007) "Parent Overweight Predicts Daughters' Increase in BMI and Disinhibited Overeating from 5 to 13 Years" Obesity 15 (6), 1544-1553.

Frankel S., C. Davison and G. Smith (1991) "Lay epidemiology and the rationality of responses to health education" British Journal of General Practice 41, 428-430.

Godfrey K. and D. Barker (2000) "Fetal nutrition and adult disease" The American Journal of Clinical Nutrition 71(suppl), 1344S-1352S.

Grimes C., L. Riddell and C. Nowson (2009) "Consumer knowledge and attitudes to salt intake and labelled salt information" Appetite 53, 189-194.

Grossman M. (1972) "On the concept of health capital and the demand for health" Journal of Political Economy 80, 223-255.

Grossman M. (2000) "The human capital model" in Handbook of Health Economics by Culyer A. and Newhouse P., Eds., North-Holland: Amsterdam, 1 (7), 348-408.

Hansen G. and E. Prescott (2002) "Malthus to Solow." American Economic Review 92, 1205-1217.

Herlihy D. (1997) The Black Death and the transformation of the West, Harvard University Press: Cambridge. 
Hill J. and J. Peters (1998) "Environmental contributions to the obesity epidemic" Science 280, 1371-1374.

Inspection Générale des Finances (IGF) (2008) Rapport sur la Pertinence et la Faisabilité d'une Taxe Nutritionelle, Documentation Française: Paris.

Jacobson M. and K. Brownell (2000) "Small taxes on soft drinks and snack foods to promote health" American Journal of Public Health 90 (6), 855-857.

Jahnke D. and P. Warschburger (2008) "Familial Transmission of Eating Behaviors in Preschool-aged Children" Obesity 16 (8), 1821-1825.

John A. and R. Pecchenino (1994) "An overlapping generations model of growth and the environment" Economic Journal 104, 1393-1410.

Kermack W. and A. McKendrick (1927) "A Contribution to the Mathematical Theory of Epidemics" Proceedings of the Royal Society of London, Series A, $115,700-721$.

Kermack W. and A. McKendrick (1932) "A Contribution to the Mathematical Theory of Epidemics. II. The problem of Endemicity" Proceedings of the Royal Society of London, Series A, 138, 55-83.

Kermack W. and A. McKendrick (1933) "A Contribution to the Mathematical Theory of Epidemics. III. Further Studies of the problem of Endemicity" Proceedings of the Royal Society of London, Series A, 141, 94-122.

Kolodinsky J., J. Harvey-Berino, L. Berlin, R. Johnson and T. Reynolds (2007) "Knowledge of current dietary guidelines and food choice by college students: better eaters have higher knowledge of dietary guidance" Journal of the american Dietetic Association 107 (8), 1409-1413.

Kuznetsov Y. and C. Piccardi (1994) "Bifurcation analysis of periodic SEIR and SIR epidemic models" Journal of Mathematical Biology, 32, 109-121.

Lake J., C. Power and T. Cole (1997) "Child to adult body mass index in the 1958 British birth cohort: associations with parental obesity" Archives of Disease in Childhood 77, 376-381. 
Lave L. and P. Seskin (1971) "Health and air pollution. The effect of occupation mix" The Swedish Journal of Economics 73 (1), 76-95.

Lindeboom M., A. Llena-Nozal and B. van der Klaauw (2009) "Parental education and child health: Evidence from a schooling reform" Journal of Health Economics 28, 109-131.

Ludwig D., K. Peterson and S. Gortmaker (2001) "Relation between consumption of sugar-sweetened drinks and childhood obesity: a prospective, observational analysis" The Lancet 357, 505-508.

Makowske M. and R. Feinman (2005) "Nutrition education: a questionnaire for assessment and teaching" Nutrition Journal 4:2.

Mamun A., D. Lawlor, R. Alati, M. O'Callaghan, G. Williams and J. Najman (2006) "Does Maternal Smoking during Pregnancy Have a Direct Effect on Future Offspring Obesity? Evidence from a Prospective Birth Cohort Study" American Journal of Epidemiology 164 (4), 317-325.

Mariani F., A. Pérez-Barahona and N. Raffin (2010) "Life expectancy and the environment" Journal of Economic Dynamics and Control, 34 (4), 798-815.

McDonald S. and J. Roberts (2006) "Aids and economic growth: A human capital approach" Journal of Development Economics 80, 228-250.

Mendez M., M. Torrent, C. Ferrer, N. Ribas-Fito and J. Sunyer (2008) "Maternal smoking very early in pregnancy is related to child overweight at age 5-7 y". The American Journal of Clinical Nutrition 87, 1906-1913.

Mitchell J. (1990) "The effect of chronic disease on work behavior over the life cycle" Southern Economic Journal 56 (4), 928-942.

Montgomery S. and A. Ekbom (2002) "Smoking during pregnancy and diabetes mellitus in a British longitudinal birth cohort" British Medical Journal 324, $26-27$.

Mytton O., A. Gray, M. Rayner and H. Rutter (2007) "Could targeted food taxes improve health?" Journal of Epidemiology and Community Health 61, 689-694. 
Osmond C. and D. Barker (2000) "Fetal, Infant, and Childhood Growth Are Predictors of Coronary Heart Disease, Diabetes, and Hypertension in Adult Men and Women" Environmental Health Perspectives 108 (3), 545-553.

Philipson T. (2000) "Economic Epidemiology and Infectious Diseases" in Handbook of Health Economics by Cuyler A. and J. Newhouse, Eds., Elsevier B.V.: Amsterdam, 1B, 1761-1799.

Pollard C., M. Miller, R. Woodman, R. Meng and C. Binns (2009) "Changes in knowledge, beliefs, and behavior related to fruit and vegetable consumption among western Australian adults from 1995 to 2004" American Journal of Public Health 99, No.2, 355-361.

Powell L. and F. Chaloupka (2005) "Parents, public policy and young smoking" Journal of Policy Analysis and Management, 24(1), 93-112.

Powers S., L. Chamberlin, K. van Schaick, S. Sherman and R. Whitaker (2006) "Maternal Feeding Strategies, Child Eating Behaviors, and Child BMI in Low-Income African-American Preschoolers" Obesity 14 (11), 2026-2033.

Revicki D. and R. Israel (1986) "Relationship between body mass indices and measures of body adiposity" American Journal of Public Health, 76 (8), 992-94.

Rudd Report (2009) Soft Drink Taxes: A Policy Brief., Rudd Center for Food Policy \& Obesity. Yale University: New Haven.

Sacerdote B. (2007) "How Large Are the Effects from Changes in Family Environment? A Study of Korean American Adoptees" Quarterly Journal of Economics, 122 (1), 119-157.

Schwartz J. and C. Byrd-Bredbenner (2005) "Young adults' knowledge of standard serving sizes and their ability to estimate servings in typical portions" Journal of the American Dietetic Association 105 (8), Supplement, 59-59.

Silventoinen K. (2003) "Determinants of Variation in Adult Body Height" Journal of Biosocial Science 35, 263-285.

Steckel R. (1995) "Stature and the standard of living" Journal of Economic Literature 33, 1903-1940. 
Steckel R. (2008) "Biological Measures of the standard of living" Journal of Economic Perspectives 22, 129-152.

Suhrcke M., R. Nugent, D. Stuckler and L. Rocco (2006). Chronic diseasse: An economic perspective, Oxford Health Alliance: London.

Vartanian L., M. Schwartz and K. Brownell (2007) "Effects of Soft Drink Consumption on Nutrition and Health: A Systematic Review and MetaAnalysis" American Journal of Public Health 97 (4), 667-675.

Vereecken C. and L. Maes (2010) "Young Chidren's dietary habits and associations with the mother's nutritional knowledge and attitudes" Appetite 54, $44-51$.

Victoria C.G., L. Adair, C. Fall, P. C. Hallal, R. Martorell, L. Richter, H. S. Sachdev (2008) "Maternal and child undernutrition: consequences for adult health and human capital" The Lancet 371, 340-357.

Wardle J., S. Sanderson, C. Guthrie, L. Rapoport and R. Plomin (2002) "Parental Feeding Style and the Intergenerational Transmission of Obesity Risk" Obesity Research 10 (6), 453-462.

WHO (2004) "WHO expert consultation. Appropriate body-mass index for Asian populations and its implications for policy and intervention strategies" The Lancet 363 (9403), 157-163.

WHO (2005) Preventing Chronic Diseases - a vital investment, Geneva: WHO Press, Department of Chronic Diseases and Health Promotion, World Health Organization.

WHO (2008) 2008-2013 action plan for the global strategy for the prevention and control of noncommunicable diseases. Geneva: WHO Press, World Health Organization.

WHO (2009) Global Infobase, https://apps.who.int/infobase/comparestart.aspx accessed on November 1, 2009.

Young A. (2005) "The gift of the dying: the tragedy of aids and the welfare of future african generation" Quarterly Journal of Economics 120, 243-266. 
Yuill T. and M. Miller (2008) "Dose-response relationship" in Encyclopedia of Earth by Cleveland, C.J., Eds., Environmental Information Coalition, National Council for Science and the Environment: Washington, D.C., http://www.eoearth.org/article/Dose-response_relationship.

Zeller M., J. Reiter-Purtill, A. Modi, J. Gutzwiller, K. Vannatta and W. Davies (2007) "Controlled Study of Critical Parent and Family Factors in the Obesigenic Environment" Obesity 15 (1), 126-136. 